\title{
Association of weight gain and metabolic syndrome in patient taking Clozapine: a 8-year cohort study
}

\author{
Ya Mei Bai ${ }^{1,2^{*}}$, Chao-Cheng Lin ${ }^{3,4}$, Jen-Yeu Chen ${ }^{5}$, Tzu Ting Chen ${ }^{5}$, Tung-Pine Su ${ }^{1,2}$ \\ From $1^{\text {st }}$ International Congress on Neurobiology and Clinical Psychopharmacology and European \\ Psychiatric Association Conference on Treatment Guidance \\ Thessaloniki, Greece. 19-22 November 2009
}

\section{Background}

Metabolic syndrome is an important side effect associated with clozapine. It has been hypothesized that weight gain contributes to the development of metabolic syndrome, but a direct diabetogenetic effect has also been suggested. We conducted an 8-year cohort study to determine the association between weight gain and metabolic parameters among schizophrenic patients on clozapine.

\section{Materials and methods}

The subjects were hospitalized schizophrenic patients who began to receive clozapine and subsequently had monthly body weight monitoring during the entire study period. Chart reviews were conducted to obtain gender, age at initiation of clozapine treatment, baseline Body Mass Index (BMI), BMI changes after the initiation of clozapine treatment, treatment duration with clozapine and concomitant psychotropic medications. Anthropometric and biochemical measurements were performed to determine the presence of metabolic syndrome.

\section{Results}

Patients were maintained on clozapine for an average treatment duration of $56.0 \pm 27.8$ (range 5 to 96) months. The prevalence of metabolic syndrome was $28.7 \%$. The cohort regression models showed that baseline BMI $(\mathrm{p}<0.0001)$ and BMI change after clozapine treatment $(\mathrm{p}<0.0001)$ were significant factors for metabolic syndrome as were most metabolic parameters except hyperglycemia and diabetes mellitus, which were related to treatment duration $(\mathrm{p}<0.05)$.

'Department of Psychiatry, Taipei Veterans General Hospital, Taipei, Taiwan

\section{Conclusions}

For patients treated with clozapine, metabolic syndrome and most metabolic parameters were related to weight gain; however, glucose dysregulation was associated with treatment duration independent of weight gain. The results confirm that monitoring body weight is important, but periodic monitoring of blood sugar may also be required for clozapine patients who do not have significant weight gain.

\section{Author details}

${ }^{1}$ Department of Psychiatry, Taipei Veterans General Hospital, Taipei, Taiwan. ${ }^{2}$ Department of Psychiatry, Collage of Medicine, National Yang-Ming University, Taipei, Taiwan. ${ }^{3}$ Department of Psychiatry, National Taiwan University Hospital, Taipei, Taiwan. ${ }^{4}$ Graduate Institute of Medical Informatics, Taipei Medical University, Taiwan. ${ }^{5}$ Department of Psychiatry, Yu-Li Veterans Hospital, Taiwan.

Published: 22 April 2010

\section{References}

1. Henderson DC, Cagliero E, Copeland PM, et al: Glucose metabolism in patients with schizophrenia treated with atypical antipsychotic agents: a frequently sampled intravenous glucose tolerance test and minimal model analysis. Arch Gen Psychiatry 2005, 62:19-28.

2. Henderson DC, Copeland PM, Borba CP, et al: Glucose metabolism in patients with schizophrenia treated with olanzapine or quetiapine: a frequently sampled intravenous glucose tolerance test and minimal model analysis. J Clin Psychiatry 2006, 67:789-797.

3. Howes OD, Bhatnagar A, Gaughran FP, et al: A prospective study of impairment in glucose control caused by clozapine without changes in insulin resistance. Am J Psychiatry 2004, 161:361-363.

4. Lamberti JS, Costea GO, Olson D, et al: Diabetes mellitus among outpatients receiving clozapine: prevalence and clinical-demographic correlates. J Clin Psychiatry 2005, 66:900-906.

doi:10.1186/1744-859X-9-S1-S132

Cite this article as: Bai et al:: Association of weight gain and metabolic syndrome in patient taking Clozapine: a 8-year cohort study. Annals of General Psychiatry 2010 9(Suppl 1):S132. 